\title{
GÉNÉRATION DU MILLÉNAIRE ET L'INFLUENCE DES RÉSEAUX SOCIAUX SUR L'EXERCICE DE LA DÉMOCRATIE : L'EXEMPLE DE L'AFRIQUE ET DU CONGO
}

\author{
Author(s) / Auteur(s) : \\ Alphonse Christian IVINZA LEPAPA \\ Docteur en Sciences de Gestion (ULB, Solvay) ; \\ Professeur Honoraire de Gestion de la FWB (Belgique) ; \\ Professeur des Systèmes d'Information de Gestion (MIS) et de Télématique de l'Enseignement Supérieur et \\ Universitaire (ESU), RDC \\ ivinza@hotmail.com
}

\begin{abstract}
Résumé :
Le concept des réseaux sociaux dans le langage courant se confond avec l'expression "réseau social" qui est utilisée pour parler des "médias sociaux". Un réseau social est un ensemble d'individus ou d'organisations reliés par des interactions sociales régulières. L'expression "médias sociaux" recouvre les différentes activités qui intègrent: la technologie, l'interaction sociale (entre individus ou groupes d'individus) et la création de contenu. Les médias sociaux utilisent l'intelligence collective dans un esprit de collaboration en ligne. Grâce à ces médias sociaux (moyens de communication sociale), les individus ou les groupes d'individus qui collaborent créent ensemble du contenu web, organisent ce contenu, indexent, modifient, commentent et combinent le contenu avec des créations personnelles.

Quelles sont les générations intéressées par ces réseaux sociaux?

Point commun elles se reconnaissent sur le même fond : le paradigme digital (l'importance à accorder aux phénomènes numériques). Cela explique l'engouement aux réseaux sociaux entre les différentes générations (grand-père, grande-mère, père, mère, enfants, milieux académique et universitaire, milieux ruraux, milieux citadins) qui se servent des mêmes outils.

Quelle est l'influence des réseaux sociaux dans la démocratie en Afrique et au RD Congo?

Les natifs de la génération Internet et du Millénaire vivent l'ère du numérique (ordinateur, baladeur, Gsm) et seraient les plus instruits de l'histoire humaine.

Les Africains et les congolais actuels sont tous enfants de ces deux générations, ils vivent l'ère du numérique (ordinateur, baladeur, Gsm) et devront être parmi les plus instruits de notre pays : ils sont nés dans la société du savoir et ils sont marqués par l'Internet comme les autres enfants du monde.

En raison de la mondialisation des Technologies de l'Information et de la communication (TIC) qui empêcherait l'usage de la liberté des échanges d'information dans les milieux africains.
\end{abstract}

\section{Keywords / Mots-clés :}

réseaux sociaux, réseau social, médias sociaux, paradigme digital, génération internet, génération du millénaire, ère du numérique 
Génération du millénaire et l'influence des réseaux sociaux sur l'exercice de la démocratie : "L'exemple de l'Afrique et du Congo"

Dans cet article, nous présentons d'abord le concept de réseaux sociaux et les termes voisins de médias sociaux. Ensuite, nous résumons un découpage historique des générations. Les générations Internet et du Millénaire sont celles du savoir numérique. L'influence des réseaux sociaux sur la démocratie en Afrique est né du printemps arabe avec l'apparition du jeune rebelle numérique.

Les congolais actuels font partie des générations du savoir et n'échappe pas au paradigme digital.

\section{Concept des Réseaux sociaux ${ }^{1}$}

\section{Introduction}

Un réseau social est un ensemble d'individus ou d'organisations reliés par des interactions sociales régulières.

Du point de vue académique, l'analyse des réseaux sociaux, se base sur

- la théorie des réseaux,

- l'usage des graphes,

- et l'analyse sociologique.

L'expression réseau social a été introduit par l'anthropologue australien John Arundel Barnes en 1954.

\section{Concepts voisins}

Cependant, dans le langage courant, l'expression "réseau social" est utilisée pour parler des "médias sociaux".

L'expression "médias sociaux" recouvre les différentes activités qui intègrent

- la technologie,

- l'interaction sociale (entre individus ou groupes d'individus),

- et la création de contenu.

\section{Médias Sociaux, point de vue informatique}

Andreas Kaplan et Michael Haenlein définissent les médias sociaux comme

"un groupe d'applications en ligne

- qui se fondent sur la philosophie et la technologie du net

- et permettent la création et l'échange du contenu généré par les utilisateurs". ${ }^{2}$

En d'autres termes, il s'agit d'un ensemble des applications en ligne permettant à l'internaute de s'inscrire, de se créer une identité virtuelle et de se constituer un réseau de connaissances personnelles et/ou professionnelles.

\section{Intelligence du groupe pour les médias sociaux}

Les médias sociaux utilisent l'intelligence collective dans un esprit de collaboration en ligne.

Grâce à ces médias sociaux (moyens de communication sociale), les individus ou les groupes d'individus qui :

- collaborent et créent ensemble du contenu web,

- organisent ce contenu,

- indexent, modifient, commentent et combinent le contenu avec des créations personnelles.

\footnotetext{
${ }^{1}$ https://fr.wikipedia.org/wiki/R\%C3\%A9seau_social

2 https://fr.wikipedia.org/wiki/R\%C3\%A9seau_social
} 
En d'autres termes, un réseau social fournit des outils de communication, d'échanges, de création et de partage d'informations ludiques ou professionnelles liées à l'actualité ou à un domaine d'expertise spécifique.

\section{Les techniques}

Les médias sociaux utilisent de nombreuses techniques parmi lesquelles, le web, les blogues, les wikis, le partage de photos,

- le vidéo-partage (YouTube), l'organisation de sorties amicales,

- les mondes virtuels, les micro blogues,

- $\quad$ 'avènement de 03B ?

- etc.

\section{Du Web au Web 2.0}

L'émergence des réseaux sociaux est liée aux révolutions technologiques et techniques :

- L'apparition de la technologie

- $\quad$ AJAX (JavaScript + XML) a permis des interactions plus rapides avec les pages Internet.

De ce fait, le nombre de membres de ces réseaux sociaux s'est allongé. D'une part car les interactions étant plus rapides, consulter Internet est devenu plus confortable. Mais d'autre part, car les utilisateurs prennent conscience de leur pouvoir d'interagir sur la toile. C'est ce qui a donné naissance au Web 2.0.

\section{Liste des réseaux sociaux ${ }^{3}$}

Depuis quelques années, les réseaux sociaux sont devenus des outils incontournables sur Internet.

Nous citerons 10 réseaux sociaux qui ont dépassé un million d'utilisateurs actifs mensuels et expliciterons les trois premiers.

1. Facebook : 1,23 milliard d'utilisateurs

Facebook est aujourd'hui le seul réseau social qui dépasse le milliard. Sa croissance est encore forte malgré son âge (plus de 10 ans), notamment grâce à ceux qui accèdent à Facebook via mobile.

2. Tencent QQ : 798 millions d'utilisateurs

Tencent QQ n'a rien à voir avec Linux. Le service web chinois permet d'envoyer facilement des messages instantanés. En Asie, Tencent QQ est bien plus populaire que Skype !

3. Tencent Ozone : 623 millions d'utilisateurs

En 2005, l'entreprise Tencent lance Qzone, son nouveau réseau social.

Il permet de créer un blog, partager des infos et des photos, écouter de la musique et regarder des vidéos. Toute ressemblance avec un réseau social de Palo Alto ne peut être que fortuite.

4. Google+ : 359 millions d'utilisateurs

5. Skype : 280 millions d'utilisateurs

6. Twitter : 241 millions d'utilisateurs

7. RenRen : 194 millions d'utilisateurs

8. line : 202 millions d'utilisateurs

8. Linkedln : 187 millions d'utilisateurs

9. Instagram : 150 millions d'utilisateurs

10. Nimbuzz : 150 millions d'utilisateurs.

\footnotetext{
${ }^{3}$ COEFFE Thomas, 15 Réseaux sociaux qui ont dépassé les 100 millions d'utilisateurs, $\mathrm{http}: / / \mathrm{www}$. blogdumoderateur.com/reseaux-sociaux-100-millions/
} 


\section{Quelles générations pour les réseaux sociaux}

"Selon Howe et Strauss (2000), une nouvelle génération naît approximativement tous les 20 ans. Alors que certains traits, valeurs, attitudes et comportements sont communs à plus d'une génération, il est reconnu que chaque génération possède un nombre important de caractéristiques qui lui sont propres." 4

\section{Un découpage historique des générations}

En nous inspirant du découpage de Howe et Strauss présenté par Guy PARE, nous considérons aujourd'hui qu'il a existé six générations. Et nous pouvons même renchérir qu'une septième génération serait naissante à partir de 2017.

Tableau 1. Découpage historique des générations

\begin{tabular}{|l|l|}
\hline Période de naissance & Découpage historique des générations \\
\hline Avant 1925 & Traditionalistes bâtisseurs \\
\hline $1925-1946$ & Traditionalistes silencieux \\
\hline $1947-1964$ & Baby - Boomers \\
\hline $1965-1976$ & Génération X \\
\hline $1977-1997$ & Génération Y ou Internet \\
\hline Après 1997 & Génération du millénaire \\
\hline A partir de 2017 & $\boldsymbol{?}$ \\
\hline
\end{tabular}

La première génération va de l'année 1901 jusque 1925, elle est formée des traditionalistes bâtisseurs. "Au retour de la Première Guerre mondiale, ils se sont mis à la tâche d'édifier une société nouvelle. Ils ont survécu à la Grande Dépression et ont entrepris de reconstruire la société. Puis, ils sont allés faire la Deuxième Guerre mondiale et, au retour, ont construit l'économie, les villes, les autoroutes, les chemins de fer et les lignes aériennes (.......). .La force de caractère des membres de cette génération a été le moteur de son succès et de sa longévité. Discipline, oubli de soi, esprit d'équipe et volonté de contribuer au bien commun : voilà les valeurs fondamentales qui ont guidé cette génération." 5

La deuxième génération est formée des traditionalistes silencieux, sont les personnes nées entre 1925 et 1946. "Les traditionalistes silencieux sont des enfants très protégés qui ont grandi en voyant leurs parents faire de lourds sacrifices pour eux. Ce groupe a regardé faire les bâtisseurs et s'est identifié à leurs valeur.» ${ }^{6}$

Ces deux premières générations sont caractérisées par le respect des valeurs traditionnelles, d'où ils tirent l'appellation de traditionnalistes.

La troisième génération est appelée "les baby-boomers" ce sont des personnes nées entre 1947 et 1964. Cette période est associée à une prospérité de la reprise économique de l'après -guerre. Cette prospérité a conduit "à l'optimisme et à de grands espoirs ayant incité les traditionalistes à avoir plusieurs enfants". ${ }^{7}$

"Un large fossé s'est creusé entre les valeurs, les attitudes et les actes des baby-boomers et ceux de leurs parents (traditionalistes). Parmi les raisons ayant favorisé le développement de ce fossé, on

\footnotetext{
4 PARE Guy, La Génération Internet : un nouveau profil d'employés, in Revue internationale de Gestion, volume 27, numéro 2, Eté 2002, pages 46-53.

5 PARE Guy, op. cit., pages 46-53.

6 PARE Guy, La Génération Internet : un nouveau profil d'employés, in Revue internationale de Gestion, volume 27, numéro 2, Eté 2002, pages 46-53.

7 PARE Guy, op.cit., pages 46-53.
} 
trouve les nouvelles méthodes d'éducation, l'influence de la télévision et, surtout, la disparité de niveaux de scolarité." 8

La quatrième est appelée "génération $X$ ", elle est constituée des personnes qui sont nées entre 1965 et 1976. "Il s'agit d'une génération charnière qui a subi le passage de l'économie industrielle à la société du savoir (Cartier, 1999). À cet égard, elle vit les problèmes causés par la génération précédente : endettement individuel et national (obligation d'atteindre le déficit zéro, par exemple), détérioration de l'environnement urbain, rationalisation (downsizing) des entreprises, précarité des emplois, etc." 910

La cinquième génération est appelée génération Internet, elle est constituée des personnes nées entre 1977 et 1997. Leurs parents sont en majorité issus de la génération des baby-boomers; car, ils sont nés avec le savoir. Les anglo-saxons appellent cette génération Y, Digital natives, Net Generation, E - Generation ou Generation.com. ${ }^{11}$

De même que la télévision a marqué l'éducation des baby-boomers, aujourd'hui l'internet est l'outil principal de cette génération. "Les enfants et les adolescents ont aujourd'hui recours à l'informatique dans une grande variété d'activités. Par exemple, ils utilisent des ordinateurs, de nombreux logiciels et surtout Internet pour étudier et faire leurs devoirs, pour se divertir en jouant à des jeux, en participant à des discussions en ligne et/ou en téléchargeant de la musique sur le Web, pour s'informer ou faire de la recherche en ligne sur des sujets qui les passionnent, pour conseiller leurs parents lorsqu'ils font des achats en ligne et pour gérer leurs finances personnelles, pour ne nommer que ces activités-là." ${ }^{12}$

Les natifs de la sixième génération, celle du millénaire comporte les germes de la génération Internet ou $\mathbf{Y}$ et véhiculent les mêmes caractéristiques. Est-ce un prolongement de la génération précédente ? Howe et Strauss (2000) les appellent "Millennials". ${ }^{13}$

\section{Génération Internet (Y) et du Millénaire}

Les enfants de la génération Internet $(\mathrm{Y})$ et du Millénaire vivent l'ère du numérique (ordinateur, baladeur, Gsm) et seront les plus instruits de l'histoire humaine. Ils sont nés dans la société du savoir :

- ils sont marqués par l'internet (comme les "baby boomers" l'étaient par la télévision),

- en raison de la mondialisation des Technologies de l'Information (internet) les enfants et les adolescents du monde se ressemblent de plus en plus (ils ont une culture homogène).

La culture Internet "découle de l'utilisation des médias numériques et interactifs. Nous devons tous ouvrir l'oeil, rappelle Tapscott (1998), car la culture qui naît de l'expérience du cyberespace est annonciatrice de la culture que créeront ces jeunes demain, lorsqu'ils seront devenus les leaders de notre société et de nos organisations." ${ }^{14}$

"À bien des égards, les jeunes de la génération Internet créent des collectivités de toutes sortes sur la Toile. Certains membres de ces collectivités sont des jeunes que l'on a rencontrés à l'école et que l'on fréquente en chair et en os, mais on constate que de plus en plus ces collectivités font place à d'autres membres. Ces communautés virtuelles aident les enfants à acquérir de la maturité car elles les obligent à se forger des valeurs, à faire preuve de jugement, à analyser, à évaluer, à critiquer et même à venir en aide à un autre. Cette interaction encourage la confiance en soi, bien que souvent on fasse appel au cybergroupe pour trouver du soutien émotionnel (Tapscott, 1998)." 15

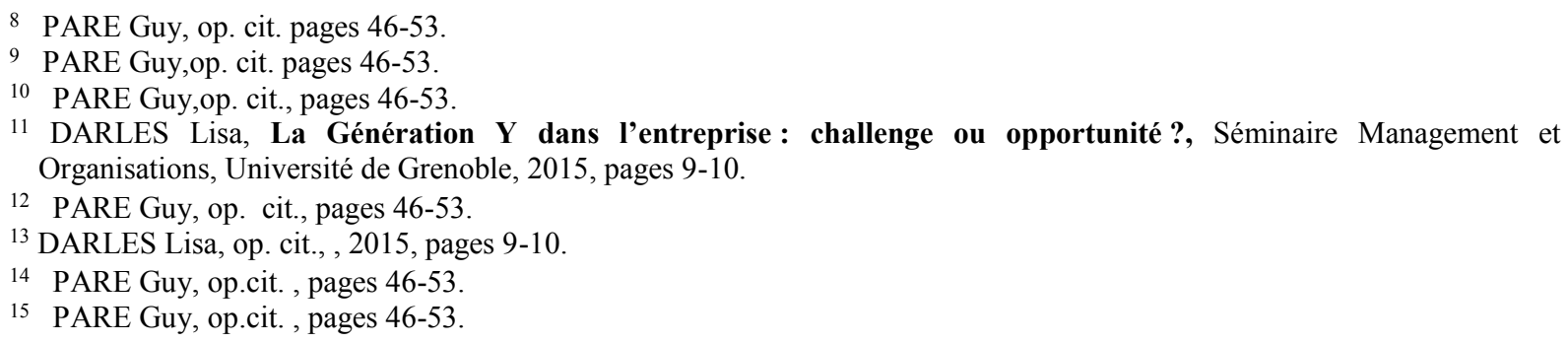




\section{Le point commun}

Comment expliquer cet engouement aux réseaux sociaux entre les différentes générations (grand-père, grande -mère, enfants) qui se servent des mêmes outils (peut être pas de la même façon)?

Point commun : Ils se reconnaissent sur le même fond : le paradigme digital (l'importance à accorder aux phénomènes numériques).

"En raison de la mondialisation des technologies de l'information, et en particulier d'Internet, on constate que les enfants et les adolescents du monde se ressemblent de plus en plus. Leur culture est homogène : ils aiment les sensations fortes, les nouvelles expériences et les voyages. Ils veulent profiter de tout immédiatement. Leur accès à la connaissance est marqué par la globalité et l'instantanéité." 16

"Ils vivent dans un environnement beaucoup plus multiculturel et plurilingue, acceptant le fait qu'il puisse exister plusieurs façons de vivre (Cartier, 1999)." 17

Il y a une révolution à l'oeuvre dans la génération Internet. La mission de cette génération ne "sera pas de raser les vieilles institutions qui ne marchent plus, mais d'en construire de nouvelles qui marchent (Tapscott, 1998; Howe et Strauss, 2000)." 18

\section{Quel réseau social? Pour qui ?}

Tous les réseaux sociaux visent à répandre le savoir de l'ère numérique

- à la génération Internet (enfants nés entre 1977 et 1997),

- et à la génération du millénaire (ceux qui naissent après 1997).

\section{L'influence des réseaux sociaux sur l'exercice de la démocratie en Afrique}

Les Africains et les congolais actuels sont en majorité, issus de ces deux générations, ils vivent l'ère du numérique (ordinateur, baladeur, Gsm) et devront être parmi les plus instruits de notre continent.

\section{L'influence des réseaux sociaux sur l'exercice de la démocratie en Afrique}

L'influence exemplaire des réseaux sociaux sur la démocratie en Afrique est celle des militants arabes sur Internet qui a abouti à des manifestations qui ont conduit à la chute de Ben Ali en Tunisie et de Moubarak en Egypte. C'était la première découverte de l'activisme démocratique en ligne dans le monde arabe que l'on caractérise par son fanatisme religieux et son arriération technologique.

Les soulèvements après les élections iraniennes de 2009 - ont été relayés partout dans le monde à travers Twitter. Sa réussite était certaine dans la mesure où, en Egypte, l'appel à la grève générale lancé sur les réseaux sociaux le 6 avril 2008 gardait toute son effervescence.

"A la faveur des événements en Tunisie puis en Egypte, sans oublier les autres foyers de protestation qui se multipliaient à l'époque, de la Péninsule arabe (Oman, Bahreïn, Yémen...) à l'extrémité marocaine du Maghreb, on a vu par conséquent surgir la figure inédite du "jeune rebelle arabe" dont le portrait - doté de caractéristiques ambiguës, comme une sorte d'hybride."19

"Un jeune rebelle, produit par une génération qu'on disait pourtant perdue pour la politique, et dont la personnalité composite s'exprimait à travers une panoplie d'outils numériques (tablettes, réseaux sociaux, logiciels de montage, palette graphique...), alliés à une détestation (supposée) des pratiques extrémistes de l'islam."'20

\footnotetext{
16 PARE Guy, op.cit., pages 46-53.

17 PARE Guy, op.cit., pages 46-53.

18 PARE Guy, op.cit. , pages 46-53.

${ }^{19}$ GONZALEZ-QUIJANOYves, "Internet, le "Printemps arabe " et la dévaluation du cyberactivisme arabe ", Égypte/Monde arabe, Troisième série, 12 | 2015, mis en ligne le 25 mars 2015, consulté le 14 septembre 2016. URL : http://ema.revues.org/3400

${ }^{20}$ GONZALEZ-QUIJANOYves,op.cit.
} 
"L'entrée en scène des jeunes rebelles du numérique accompagnait une mise en récit totalement inédite pour la région du "Printemps arabe", désormais appréhendé comme la "première révolution du troisième millénaire", une révolution témoignant, par son importance, de la capacité du numérique à produire des changements politiques radicaux. Sous le vent de folie qui balayait le monde arabe, avec la chute de deux longues dictatures et les inquiétudes de plus d'un régime dans la région, les scénarios ne s'intéressaient plus aux acteurs politiques traditionnels."21

\section{L'influence des réseaux sociaux sur l'exercice de la démocratie au Congo}

\section{Génération Internet et du Millénaire au Congo}

Les natifs de la génération Internet et du Millénaire vivent l'ère du numérique (ordinateur, baladeur, Gsm) et seraient les plus instruits de l'histoire humaine.

- Ils sont nés dans la société du savoir comme les autres enfants du monde,

- Ils sont marqués par l'internet comme les autres enfants du monde.

En raison de la mondialisation des Technologies de l'Information et de la communication (TIC), qui empêcherait l'usage de la liberté des échanges d'information dans les milieux africains.

\section{Problématique de l'influence des réseaux sociaux sur la démocratie en au Congo}

Les natifs des générations Internet et du Millénaire sont très indépendants et autonomes. Ce trait de caractère provient du fait qu'ils sont des chercheurs actifs d'informations et non pas des capteurs passifs. "Leur accès sans précédent à l'information leur donne aussi49la capacité d'acquérir le savoir nécessaire pour vérifier les informations qui pourraient leur sembler erronées."

Le fait que ces deux générations soient indépendants, autonomes et cherchent la vérité dérangent souvent le pouvoir en place. C'est pourquoi au Congo, pour brouiller certaines informations, l'autorité ordonne la coupure des connexions internet parfois pendant toute une semaine. Cela s'observe aussi pour les chaînes de télévision turbulente qui disent trop vraies pour lesquelles on suspend la fréquence parfois pendant plusieurs mois.

Les TIC font évoluer ces natifs "non pas vers l'exclusion, mais vers une plus grande inclusion sociale." Internet pousse ces congolais à passer de la sphère locale à la sphère mondiale. Dans ces conditions, il est difficile d'empêcher ces générations de s'informer et d'établir la vérité, car leur migration de la sphère locale vers la sphère mondiale est irréversible.

\section{Réseaux de télécommunications au Congo}

Un "réseau de télécommunications se compose de deux éléments : un élément tangible, l'infrastructure, et un élément intangible, les services."

L'infrastructure "est formée d'un ensemble de nœuds, systèmes informatiques, reliés entre eux et aux applications des adhérents par des lignes directes de télécommunications."

Les services peuvent être fournis par des organismes de télécommunications (service de base) ou par des sociétés de services (services à valeur ajoutée).

L'usage de l'internet au Congo est très répandu dans le pays. En l'absence d'une infrastructure filaire qui est à peine en installation (fibre optique), les connexions internet sont de très mauvaise qualité. Plusieurs opérateurs réseaux de téléphonie mobile (Vodacom, Airtel, Orange), proposent des connexions Wi-Fi tant bien que mal.

\section{O3b Networks : Internet par satellite pour les pays émergents}

O3b Networks est une alternative qui propose 1'Internet par satellite pour les pays émergents. ${ }^{22} C^{\prime}$ 'est un opérateur de satellites de télécommunications. Son siège social se situe à Jersey aux USA. Mais les systèmes au sol et le développement technique sont gérés par une filiale située à Englewood (USA).

\footnotetext{
${ }^{21}$ GONZALEZ-QUIJANOYves,op.cit.

22 IVINZA LEPAPA A. C., Introduction à la Télématique et aux réseaux informatiques, Presses universitaires de Bruxelles, 2016, pages 50 et sq.
} 
$O 3 B$ est l'acronyme de Other 3 Billion, c'est-à-dire les autres trois milliards de la population mondiale non couverte par Internet. Pour répondre aux besoins des populations des pays émergents ayant un très mauvais accès à l'Internet, O3b Networks parie sur le satellite. Dès 2005, Greg Wyler, le fondateur d'O3b Networks a parié sur le satellite plutôt que sur le développement d'infrastructures terrestres, pour réduire cette fracture numérique et apporter l'Internet à bas coût à 150 pays des marchés émergents d'Asie, d'Afrique, d'Amérique latine et du Moyen- Orient.

Cette société développe une constellation de satellites Internet, construits par Thales Alenia Space. Les premiers lancements ont été opérés en ce mois de Mai 2016. Les Débits annoncés (débit de 1, 25 Gbit/s.) seront comparables à la fibre optique. O3b Networks va fournir aux opérateurs une "capacité de trunking et de connectivité pour les réseaux de télécommunications à des débits et des temps de réponse comparables à ceux de la fibre optique".

Pour utiliser les services Internet d'O3b, les utilisateurs devront souscrire à une offre de service auprès d'un opérateur télécom local (en RDC, par exemple Orange, Airtel, Vodacom, etc.) ou à un SRVA (Vodanet, Tigônet, Innet, etc.).

Le projet d'O3b Networks va déployer une constellation de satellites sur une orbite équatoriale, pour fournir un accès Internet très haut débit à bas prix, accessible au plus grand nombre d'habitants du tiers monde.

Le service d'Internet à haut débit sera délivré par une constellation de satellites en orbite moyenne dans le plan de l'équateur, à une altitude de $8063 \mathrm{~km}$, la durée de visibilité des satellites étant de 45 minutes. Cette orbite, avec une période de 288 minutes (5 révolutions par jour), permet un passage au-dessus du même point de l'équateur toutes les 6 heures, soit un déplacement d'un degré en longitude terrestre chaque minute.

Les antennes peuvent être pointées vers un point quelconque de la Terre en quelques minutes, rayonnant dans une surface de $500 \mathrm{~km}$ de diamètre. Ce type de pointage est intéressant pour les régions forestières comme celles des certaines provinces de la RDC qui ne peuvent être desservies par la fibre optique et les opérateurs mobiles avec leurs technologies actuelles.

Formulation systémique de l'influence des réseaux sociaux sur l'exercice de la démocratie

L'influence des réseaux sociaux sur l'exercice de la démocratie peut être modélisée dans la théorie des systèmes, suivant les aspects suivants (figure 2).

Ce modèle comprend sept composants ou sous-ensembles (Technologie, Génération, Contenu, Environnement, Pouvoir, Comportement des autorités politiques, Système politique), pouvant être regroupés grossièrement en quatre systèmes (système actif, système opérateur, système régulateur, système passif - voir figure 1).

Figure 1. Les composants du modèle

\begin{tabular}{|c|}
\hline $\begin{aligned} \text { Système actif } & =\text { Variables d'entrée } \\
& =\text { Variables exogènes }+ \text { variables de commande ou d'actions } \\
& =\text { Technologie, Génération, Contenu, Environnement, Pouvoir }\end{aligned}$ \\
\hline $\begin{aligned} \text { Système passif } & =\text { Variables de sortie } \\
& =\text { Système politique }(\text { démocratie })\end{aligned}$ \\
\hline Système opérateur = Comportement des autorités politiques \\
\hline $\begin{aligned} \text { Système régulateur } & =\text { Variables de commande ou d'actions } \\
& =\text { Philosophie du pouvoir }\end{aligned}$ \\
\hline
\end{tabular}

Le système actif comprend les composants Technologie, Génération, Contenu, Environnement, Pouvoir, Comportement des autorités politiques, Système politique qui constituent les variables d'entrée. Les composants Technologie, Génération, Contenu, Environnement sont constitués des variables exogènes ou contingentes, alors que le composant Pouvoir regroupe les variables de commande. 
Figure 2. Les composants du modèle d'influence des réseaux sociaux sur l'exercice de la démocratie

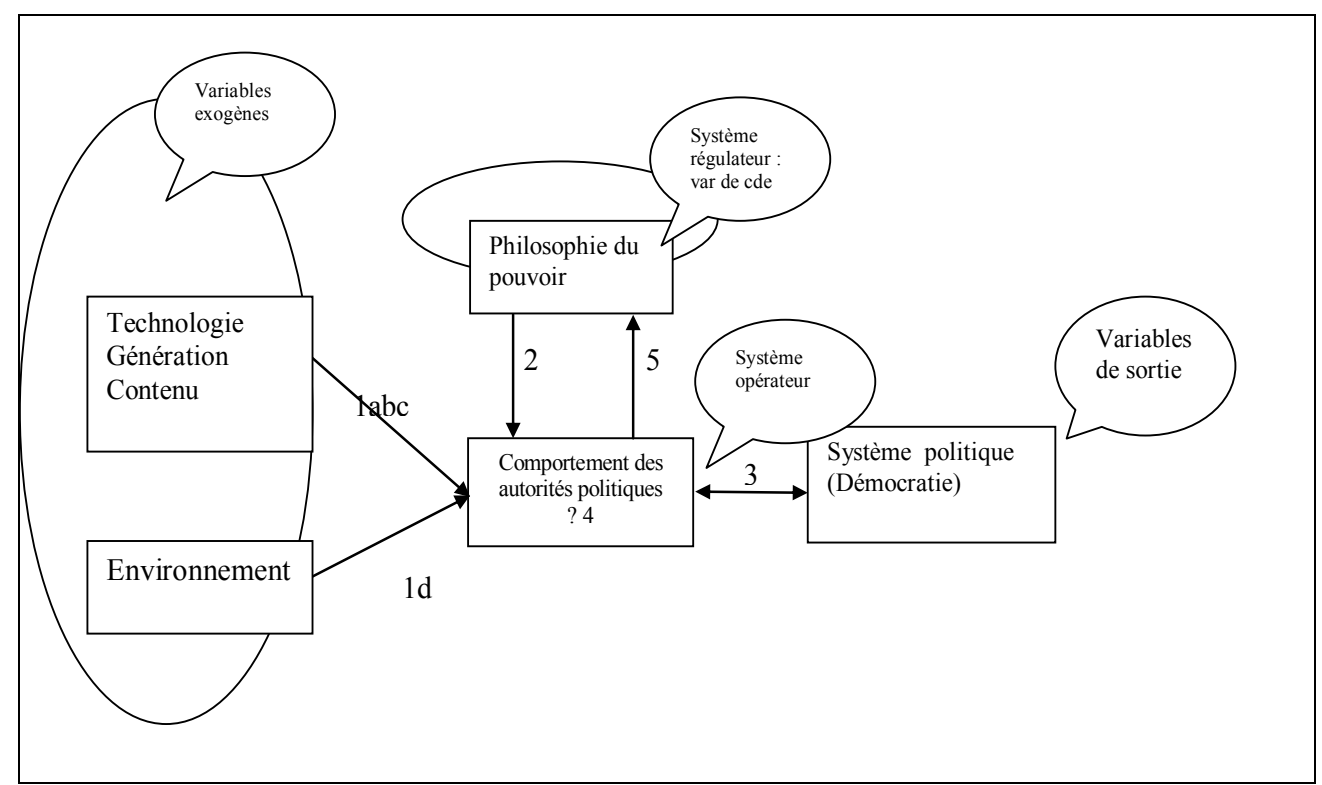

Le système opérateur est représenté dans notre modèle par le sous-ensemble (composant) comportement des autorités politiques. Il "subit les entrées et produit les sorties du système, mais il est guidé par les commandes imposées par le régulateur». ${ }^{23}$

Le système opérateur effectue une transformation qualitative des entrées sorties et fonctionne comme un système causal indéterminé (incertain).

Un système régulateur a, en général, une triple fonction informationnelle: celle de capteur, de correcteur et d'effecteur. Il est, pour des raisons de simplicité du modèle, inclus dans le composant philosophie du pouvoir.

Le système passif qui donne l'état du système à la sortie avec la variable système politique qui détermine le type de démocratie adoptée (parlementaire, présidentielle, etc.).

Le modèle d'influence des réseaux sociaux sur la démocratie utilise deux types de couplage entre les différents composants : le couplage en série et le couplage par rétroaction qui sont utilisés dans les structures caténaire et cyclique. Pour des raisons de simplicité et parce que notre modèle n'est pas normatif, il n'exploite pas totalement le système contrôleur (ou régulateur) qui se limite au rôle de correcteur.

\section{Conclusion Générale}

Depuis l'apparition du jeune rebelle numérique arabe, les pays sub sahariens qui comptent en majorité dans sa population les natifs des générations Internet et du Millénaire, n'échappent pas au paradigme digital et constitue aujourd'hui la problématique dans l'exercice de la démocratie.

Ces natifs sont indépendants, autonomes et critiques. Ils n'échappent pas à la mondialisation comme phénomène global du point de vue économique et social.

23 WALLISER Bernard, Systèmes et Modèles, Editions du Seuil, Paris, 1977, page 27. 


\section{Références}

- DARLES Lisa, La Génération Y dans l'entreprise : challenge ou opportunité ?, Séminaire Management et Organisations, Université de Grenoble, 2015.

- DUBE Line et PARE Guy, Les Technologies de l'Information et l'organisation à l'ère du virtuel employés, in Revue internationale de Gestion, volume 24, numéro 2, Eté 1999, pages 14-22.

- IVINZA LEPAPA Alphonse, " Analyse de l'introduction de l'EDI dans les entreprises congolaises : une contribution à l'impact organisationnel des TI ", Tome 1, EUE, 2010.

- IVINZA LEPAPA A. C., Introduction à la Télématique et aux réseaux informatiques, Presses universitaires de Bruxelles, 2016.

- IVINZA LEPAPA A. C., Développement Economique de Mai-Ndombe : une vision stratégique, Edition Bookelis, 2016, Paris.

- PARE Guy, La Génération Internet : un nouveau profil d'employés, in Revue internationale de Gestion, volume 27, numéro 2, Eté 2002, pages 46-53.

- Réseau social Source : https://fr.wikipedia.org/wiki/R\%C3\%A9seau_social?oldid=122591320

- COEFFE Thomas, 15 Réseaux sociaux qui ont dépassé les 100 millions d'utilisateurs, http://www.blogdumoderateur.com/reseaux-sociaux-100-millions/

- GONZALEZ-QUiJANOYves, "Internet, le "Printemps arabe" et la dévaluation du cyberactivisme arabe", Égypte/Monde arabe, Troisième série, $12 \mid 2015$, mis en ligne le 25 mars 2015, consulté le 14 septembre 2016. URL : http://ema.revues.org/3400

- WALLISER Bernard, Systèmes et Modèles : Introduction critique à l'analyse des systèmes, Editions du Seuil, Paris, 1977. 